\title{
Integration und Desintegration qua Leistung - zu den Ambivalenzen eines zentralen Anerkennungsprinzips der modernen Gesellschaft
}

\author{
Kai Dröge ${ }^{1, *}$ \\ 1 Institut für Sozialforschung, Frankfurt, Germany / Hochschule Luzern, Switzerland \\ * E-Mail: k.droege@em.uni-frankfurt.de
}

\section{Zusammenfassung}

Der Beitrag verfolgt die These, dass das Leistungsprinzip in der Geschichte der modernen Gesellschaft immer sowohl integrierende als auch desintegrierende Wirkungen entfaltet hat. Dies liegt in der Unterbestimmtheit und den Ambivalenzen der grundlegenden Idee von Leistung begründet. Zu seiner konkreten Anwendung bedarf dieses Prinzip immer der Interpretation im Kontext einer je spezifischen historischen Situation - also politischer Aushandlungen, was als sozial anerkennungswürdige Leistungen gelten soll und Integrationschancen eröffnet. Dabei werden jedoch auch blinde Flecken produziert, die Leistungsbeiträge entwerten bzw. sozial unsichtbar machen. Dies hält die gesellschaftlichen „Kämpfe um Anerkennung“ (Axel Honneth) in Gang.

Der Beitrag entfaltet diese Argumentation sowohl ideengeschichtlich-theoretisch als auch empirisch, basierend auf eigenen Forschungen zu aktuellen Deutungskonflikten rund um ,Leistung' in der Erwerbsarbeit.

\section{Schlüsselwörter}

Leistung, Leistungsprinzip, Anerkennung, Integration, Arbeit

\section{Integration, disintegration, merit - on the ambivalences of a central principle of recognition in modern society}

\begin{abstract}
This article argues that the achievement principle has always deployed socially integrating as well as disintegrating effects in modern society. This has to do with the fact that the basic idea of 'merit' is so under-determined and ambivalent in itself. In order to apply this principle in any historic situation it needs interpretation - a political bargaining about what should actually be considered as a merit or an achievement, what deserves recognition and offers chances for social integration. But this also produces blind spots, devalues certain contributions or makes them socially invisible. As a result, the "struggle for recognition" (Axel Honneth) keeps going on.

The article develops this argument from a theoretical as well as an empirical perspective. The latter is based on own research about today's conflicting interpretations of merit and achievement in the sphere of work.
\end{abstract}

\section{Keywords}

merit principle, recognition, social integration, work

The author has declared that no competing interests exist. 


\section{Einleitung}

Gerade im Leistungsprinzip einen Motor der sozialen Integration moderner Gesellschaften zu sehen, mag kontraintuitiv erscheinen. „Leistung“ ist ja zuallererst eine Legitimation für soziale Ungleichheit - spaltet also eher, als dass es eint und verbindet. Mit Verweis auf mangelnde Leistungen werden Teilhabechancen verweigert (bspw. im Bildungssystem), soziale Degradierungen begründet (etwa in der Arbeitswelt) und Tätigkeiten als sozial wertlos deklassiert (bspw. klassisch die Hausarbeit). Am oberen Ende der sozialen Statushierarchie dagegen wird selbstbewusst gefordert, dass Leistung sich (endlich) wieder lohnen müsse - womit in der Regel nichts Anderes gemeint ist, als dass man mehr vom Kuchen für sich behalten möchte (Dröge/ Neckel 20IO). Vor diesem Hintergrund lässt sich mit Recht fragen, was an der Rede von der Leistung eigentlich integrativ sein soll. ${ }^{\mathrm{I}}$

Dennoch gibt es ebenfalls gute Gründe, im Leistungsprinzip tatsächlich einen zentralen Modus der sozialen Integration in der modernen bürgerlichen Gesellschaft zu erblicken. Diese Gesellschaftsformation hat sich ja wesentlich auch dadurch von ihren historischen Vorgängern abzugrenzen versucht, dass sie die ständische Gliederung des sozialen Raums abschaffen und stattdessen die Statusordnung ganz auf das Leistungsprinzip umstellen wollte (Bolte I979; Hartfiel I977). In einer solchen Sozialordnung, die sich selbst als „Leistungsgesellschaft“ rechtfertigt und versteht, ist Integration kaum anders denkbar als unter Bezugnahme auf individuelle Leistung. ${ }^{2}$ Negativ formuliert könnte man sagen, dass jedes Individuum gewissermaßen auf Gedeih und Verderb darauf angewiesen ist, sich den Regeln der Leistungsgesellschaft zu fügen, um einen Platz in der Gesellschaft zu erlangen.

Aber eine solche Deutung, die alleine den Zwangscharakter der Integration qua Leistung hervorhebt, wäre eben-

I Ich verwende hier - abweichend von anderen Beiträgen in diesem Heft - nicht den Begriff der Inklusion, sondern den der Sozialintegration. Der Inklusionsbegriff hat in den letzten Jahren vor allem in sozial- und bildungspolitischen Debatten um die Teilhaben von Behinderten etc. eine wachsende Bedeutung erhalten (Wittig-Koppe/Bremer, Fritz, Hansen, Hartwig 20IO). Das ist jedoch nicht Thema dieses Beitrages.

Daneben gibt es eine im engeren Sinne sozialwissenschaftliche Verwendungsweise, die zumeist sehr eng mit dem Gegenpart, der Exklusion, verkoppelt ist. Diesen Dualismus von Innen und Außen, Ein- und Ausschluss berühre ich in der vorliegenden Analyse zwar teilweise, aber ein alleiniger Fokus darauf erschiene mit verkürzt. Die Integration qua Leistung ist komplex, kennt verschiedene Grade und Dimensionen, in denen eine Person integriert sein kann oder auch nicht. Vor allem aber verspricht sie mehr als bloße Teilhabe, sondern auch eine gerechte Sozialordnung unter den Inkludierten selbst. Der klassische Begriff der Sozialintegration erscheint mir eher geeignet, diese Aspekte in ihrer ganzen Breite zu umfassen.

2 Zwar kennt die bürgerliche Gesellschaft auch den von Leistung unabhängigen Status des Staatsbürgers bzw. der Staatsbürgerin, aus dem sich gewisse soziale Grundrechte ableiten. Aber wer sich allein darauf berufen kann, rangiert am unteren Ende der sozialen Statushierarchie bzw. ist eigentlich gar kein Bestandteil mehr davon - gilt als Teil einer „Unterschicht“, der sozial Deklassierten und Ausgeschlossenen (Dubet/ Lapeyronnie I994). falls $\mathrm{zu}$ einseitig. Das Leistungsprinzip formuliert normative Versprechen und Zusagen, aus denen sich auch im positiven Sinne individuelle und kollektive Ansprüche auf Integration und gesellschaftliche Wertschätzung ableiten lassen (Honneth I994). Im Kern lautet dieses Versprechen: Wer leistungsbereit und -fähig ist und einen Beitrag zum arbeitsteiligen Gemeinwesen erbringt, der erwirbt sich damit einen legitimen Anspruch auf gesellschaftliche Gegenleistungen in Form von Ansehen, sozioökomischen Status und Teilhabe am sozialen Leben. Dieser Anspruch ist im Zweifel auch öffentlich einklagbar - wenn nicht juristisch, so doch politisch (Abschnitt 2).

Die hier angedeutete Zwiespältigkeit des Leistungsprinzips als sowohl einende wie auch spaltende Kraft hat in der Vergangenheit zu sehr unterschiedlichen Einschätzungen über das integrative Potential dieses Prinzips geführt. Einige wichtige Stationen dieser Debatte werde ich unten nachzeichnen und diskutieren (Abschnitt 3). Allerdings möchte ich mich dabei weder auf eine Pro- noch auf eine Contra-Position festlegen, sondern die These verfolgen, dass das Leistungsprinzip in seiner konkreten gesellschaftlichen Auslegung immer zugleich integrierend und desintegrierend wirkt. Der zentrale Grund dafür liegt, so mein Argument, in der Abstraktheit und inhaltlichen Unterbestimmtheit der grundlegenden Idee von Leistung. In der modernen Gesellschaft gibt es nur wenige historisch invariante Kriterien dafür, welche konkreten menschlichen Tätigkeiten überhaupt in den Rang einer Leistung erhoben werden können. Hinzu kommt, dass auch diese Kernelemente des Leistungsprinzips keineswegs widerspruchsfrei und harmonisch zueinander stehen.

Die Konsequenz dieser inhaltlichen Unterbestimmtheit und internen Ambivalenz ist, dass sich dieses Prinzip nicht ohne weiteres auf eine historisch je spezifische gesellschaftliche Statusordnung anwenden lässt. Vielmehr bedarf es dazu immer einer Konkretion und Interpretation der grundlegenden Kriterien von Leistung auf den jeweiligen Kontext hin. In der historischen Realität basiert also „das Wirken des Leistungsprinzips ... auf einer Fülle ,politischer" Auseinandersetzungen und Entscheidungen, die ihm überhaupt erst konkreten Gehalt geben und es funktionsfähig machen“ (Bolte I979: 5I). Für die Frage nach der Integrationskraft dieses Prinzips ergeben sich daraus zwei wesentliche Konsequenzen:

\section{a) Kampf um Anerkennung, Kampf um Integration}

Da die jeweils etablierten konkreten gesellschaftlichen Leistungsdefinitionen immer nur eine von vielen möglichen Interpretationen des Leistungsprinzips darstellen, weist dieses Prinzip stets einen Deutungsüberschuss gegenüber diesen historisch je 
spezifischen Definitionen auf. Dieser uneingelöste Deutungsüberschuss bildet eine normative Ressource, auf die sich soziale Gruppen berufen können, wenn sie für die Anerkennung spezifischer Leistungsbeiträge kämpfen, die in den bisher sozial geltenden Leistungsdefinitionen unberücksichtigt geblieben sind oder unterbewertet waren (Honneth 1994). Dabei geht es immer auch um soziale Integration, weil hier benachteiligte gesellschaftliche Gruppen um legitime Ansprüche und gesellschaftliche Teilhabe kämpfen.

\section{b) Leistung und Politiken der Ausschließung und Missachtung}

Allerdings werden die gesellschaftlichen Leistungsdefinitionen durch diese Auseinandersetzungen nicht automatisch immer umfassender und inklusiver. Die internen Spannungen und Ambivalenzen dieses Prinzips haben zur Folge, dass sich neue Definitionen i.d.R. nicht einfach bruchlos als Erweiterung der alten durchsetzen lassen, sondern häufig nur auf Kosten der Entwertung vormals anerkannter Leistungsbeiträge. In der Folge ist jede historisch konkrete Auslegung des Leistungsprinzips immer auch zumindest teilweise ideologisch. Sie prämiert bestimmte Leistungsbeiträge, aber entwertet zugleich andere bzw. macht sie sozial unsichtbar. Insofern sind die sozialen Kämpfe um die gesellschaftliche Geltung bestimmter Leistungsdefinitionen immer auch von Politiken der Missachtung und Ausschließung begleitet. Der Abschnitt 3 arbeitet hierzu die theoretischen und ideengeschichtlichen Hintergründe heraus. Darauf soll in Abschnitt 4 unter Rückgriff auf eigene Forschungen zu heutigen Leistungsverständnissen in der deutschen Gegenwartsgesellschaft empirisch beispielhaft gezeigt werden, wie sich dies in konkreten Deutungskonflikten niederschlägt. ${ }^{3}$

\section{Leistung, Integration, Anerkennung}

In meiner Analyse des Verhältnisses von Leistung und sozialer Integration beziehe ich mich wesentlich auf

3 Ich werde später noch ausführen, wie schwer es ist, den Beitrag Einzelner an einem kollektiven Leistungshandeln individuell zuzurechnen. Dies gilt ebenso für den vorliegenden Text. Meine Analysen und Überlegungen sind - nicht nur in den empirischen Teilen - stark durch die mehrjährige Zusammenarbeit mit Sighard Neckel, Irene Somm und Veronika Schmid im Rahmen unserer Forschungen am Institut für Sozialforschung in Frankfurt am Main beeinflusst sowie durch die zahlreichen Diskussionen mit anderen KollegInnen, die sich in diesem Zusammenhang ergeben haben. Ihnen allen sei hier ausdrücklich gedankt. Außerdem möchte ich mich bei den OrganisatorInnen und TeilnehmerInnen des Workshops „Leistung Gestaltungsprinzip gesellschaftlicher und politischer Inklusion?“ an der Universität Wien für die Einladung und die fruchtbaren Diskussionen bedanken sowie bei den HerausgeberInnen dieses Heftes - namentlich bei Oliver Gruber - für die grandiose Unterstützung in der Erstellung dieses Beitrages. die Anerkennungstheorie von Axel Honneth (1994; 2003; 20II; 2008). Honneth geht im Anschluss an Hegel davon aus, dass Leistung eines von drei zentralen normativen Anerkennungsprinzipien der modernen Gesellschaft darstellt - neben dem Recht und den privaten Beziehungsnormen der Liebe, Freundschaft und Familie. Diese Anerkennungsprinzipien wiederum konkretisieren sich historisch in spezifischen gesellschaftlichen Institutionen - dem Berufs- und Erwerbssystem im Falle der Leistung, der Privat- und Intimsphäre im Falle der Liebe und dem Rechtssystem und der Demokratie im Falle der Anerkennung als freies und gleiches Rechtssubjekt.

Für die Frage der Integration ist wichtig, das Honneth den von Hegel kommenden Begriff der Anerkennung mit einer Konzeption von personaler Identität verknüpft, die er unter Rückgriff auf George Herbert Mead (I973) entwickelt. Mit Mead geht er davon aus, dass Identitätsentwicklung nur sozial verstanden werden kann: Um zu einem gefestigten Selbstbild zu gelangen, müssen wir uns mit der Sicht der Anderen auf uns auseinandersetzen - seien es konkrete Andere des unmittelbaren Nahbereichs oder auch dem "generalized other" (Mead), d.h. den gesellschaftlichen Normen und Verhaltenserwartungen, wie sie auch in den sozialen Institutionen der Anerkennung verkörpert sind. Die Entwicklung des Selbst und die soziale Integration sind in dieser Sichtweise unablöslich miteinander verknüpft.

Honneth geht davon aus, dass die verschiedenen Prinzipien der Anerkennung auf jeweils eigene Dimensionen in der Entwicklung unseres Selbst verweisen. In der Anerkennung unserer Leistungen erfahren wir uns als Subjekt, dessen Handeln von Bedeutung für einen größeren Sozialzusammenhang ist und durch diesen entsprechend wertgeschätzt wird. Missachtungserfahrungen dagegen können, wenn sie über längere Zeit anhalten, unser Selbst nachhaltig beschädigen. Wenn wir etwa immer wieder die Erfahrung machen, dass unsere Leistungen in Schule oder Beruf keine Anerkennung finden und mit schlechten Noten, mangelnden Aufstiegsperspektiven oder Arbeitslosigkeit beantwortet werden, so kann dies tiefgreifenden persönlichen Krisen und Empfindungen von Wert- und Nutzlosigkeit nach sich ziehen (Jahoda et al.1975). Allerdings sind Resignation und Selbstaufgabe nicht die einzig möglichen Reaktionen. Wenn das Ausbleiben von Anerkennung nicht als persönliche Schwäche, sondern als Ergebnis der Diskriminierung einer sozialen Gruppe erfahren wird, so kann sich daraus auch ein öffentlicher Kampf um Anerkennung entwickeln, der darauf zielt, die institutionalisierten Muster zu verändern. Die soziale Anerkennungsordnung insgesamt wird von Honneth somit als ein historisch fluides und wandelbares Gebilde angesehen, das in einer potentiell konflikthaften sozialen Praxis sowohl stetig reproduziert als auch verändert wird. 
Allerdings benötigen solche gesellschaftlichen Kämpfe um Anerkennung eine normative Basis. Wenn eine soziale Praxis als diskriminierend oder missachtend kritisiert wird, so wird problematisiert, dass hier eine eigentlich berechtigterweise zu erwartende Anerkennung faktisch ausbleibt. Dies aber setzt ein gewisses Vorverständnis darüber voraus, unter welchen allgemeinen Bedingungen wir davon sprechen können, dass Anerkennung, berechtigterweise‘ zu erwarten sei. Für das Leistungsprinzip bedeutet dies, dass es zumindest eine vage gesellschaftliche Vorstellung darüber geben muss, welche generellen Merkmale ,Leistungen' prinzipiell aufweisen sollten und wie eine Anerkennungsordnung grundsätzlich aufgebaut sein müsste, die auf Leistungsdifferenzen abstellt. Ohne dies wäre es kaum möglich, die je konkrete Institutionalisierung dieses Prinzips in Gestalt von Tarifvereinbarungen, Bildungstiteln oder Karrierelaufbahnen noch irgendwie als diskriminierend oder ausschließend zu kritisieren.

Honneth ist relativ zurückhaltend, was die Formulierung solcher Kernelemente des Leistungsprinzips betrifft und buchstabiert diesen Strang seiner Theorie nur begrenzt aus. Ich werde daher im Folgenden auf einen breiteren Kanon an sozialwissenschaftlicher Literatur zurückgreifen, um in Form einer ideengeschichtlichen Rekonstruktion einige wichtige Kernelemente dieses Prinzips herauszuarbeiten. Dabei geht es mir im Sinne der eingangs aufgeworfenen These insbesondere auch darum, die internen Widersprüche und Ambivalenzen auszuweisen.

\section{Leistung und Leistungsprinzip - Kern- elemente und Konflikte}

\subsection{Reziprozität, organische Solidarität, Integration}

Zunächst kann man ganz allgemein festhalten, dass das Leistungsprinzip ein Reziprozitätsverhältnis zwischen Individuum und Gesellschaft entwirft: „Jeder soll nach Maßgabe der Mühen, Kosten und Aufwendungen, die die Erstellung einer Leistung verursacht hat, durch entsprechende Gegenleistungen entschädigt werden“ (Bolte I979, I8).

Diese Reziprozitätsidee ist eng verknüpft mit den grundlegenden Funktionsmechanismen der modernen arbeitsteiligen Gesellschaft. Der oder die Einzelne bringt Güter und Dienste hervor, von denen viele Andere profitieren. Gleichzeitig ist er oder sie aber auch selbst auf die Leistungsbeiträge Anderer angewiesen. Diese wechselseitige Abhängigkeit begründet - idealerweise - ein gegenseitiges Verpflichtungsverhältnis, das Émile Durkheim (1992 [1893]) als „organische Solidarität" beschrieben hat. Damit ist auch die zentrale integrative Kraft des Leistungsprinzips benannt: Im besten Falle erlaubt es dem oder der Einzelnen, sich als integralen Bestandteil eines arbeitsteiligen Gemeinwesens zu betrachten, in der die eigene Leistung von Wert für die Gemeinschaft ist und von dieser auch entsprechend honoriert wird (Honneth 1994; Honneth 2008). Allerdings macht Durkheim ebenfalls deutlich, dass sich solche Erfahrungen von Reziprozität, Solidarität und Zugehörigkeit keineswegs zwingend einstellen müssen. Wenn die sozialmoralische Entwicklung einer Gesellschaft nicht mit ihrer wirtschaftlichen Schritt hält, wenn die Arbeitsteilung sich verändert ohne dass ein Bewusstsein darüber entsteht, wie die individuellen Leistungsbeiträge im Rahmen eines größeren Ganzen sinnvoll zusammenspielen - dann kann dies zu anomischen Tendenzen und gesellschaftlicher Desintegration führen (Durkheim I973).

\subsection{Chancengleichheit und soziale Ungleichheit}

Eine fundamentale Voraussetzung dafür, dass das Leistungsprinzip seine integrative Kraft in der Gesellschaft entfalten kann, ist Chancengleichheit. Damit ist nicht eine absolute Gleichheit der Startbedingungen gemeint, die auch die Unterschiede in den individuellen Talenten, Stärken und Schwächen völlig nivellieren müsste. Aber wenn einzelne Personen(gruppen) ganz von der Teilnahme am gesellschaftlichen Leistungswettbewerb ausgeschlossen bzw. darin systematisch benachteiligt sind, wenn sie also ihre unterschiedlichen Talente nicht mehr fruchtbar einbringen können, dann ist die Reziprozität von Leistung und Gegenleistung grundsätzlich gestört. Letztlich würde dies in eine segmentierte Gesellschaft führen, in der soziale Integration nur noch „mechanisch“ und nicht mehr „organisch“ möglich ist (Durkheim I992).

Gleichzeitig aber wirft die Forderung nach Chancengleichheit auch einige schwer lösbare Probleme auf. Das Leistungsprinzip begründet und rechtfertigt eine soziale Ungleichheitsordnung. Soziale Ungleichheit aber ist der Realisierung von Chancengleichheit in einer Gesellschaft generell eher abträglich. Phänomene wie soziale Schließung und die Vererbung von Status zwischen den Generationen behindern den freien und gleichen Wettbewerb um begehrte Positionen (Tumin 1966). Je stärker eine Gesellschaft durch soziale Ungleichheitsrelationen gekennzeichnet ist, desto schwieriger ist es, Chancengleichheit faktisch herzustellen.

Dieses Grundproblem ist charakteristisch für eine ganze Tradition der Kritik an der Leistungsgesellschaft: Sind die Institutionalisierung von sozialer Ungleichheit und die Herstellung von Chancengleichheit im gesellschaftlichen Leistungswettbewerb zwei sich wechselseitig ausschließende Prinzipien? Wie lassen sich ungleiche Startchancen der Individuen ausgleichen, ohne den Leistungswettbewerb und die motivationale Kraft des Leistungsprinzips zu stark zu beeinträchtigen? Wie lassen sich Diskriminierungen vermeiden, ohne zugleich 
zu verunmöglichen, dass sich unterschiedliche Talente auch unterschiedlich entfalten? Um diese und ähnliche Fragen wurde und wird immer wieder heftig gestritten - klassisch etwa mit Blick auf das Bildungssystem (Bourdieu/Passeron 197I), aktuell beispielsweise in der Debatte um Frauenquoten in den Führungspositionen von Wirtschaft und Politik.

Hier wird ein zentrales Problem erkennbar, das jede konkrete gesellschaftliche Auslegung und Anwendung des Leistungsprinzips irgendwie lösen muss. Auf der einen Seite entwirft und legitimiert dieses Prinzip eine soziale Ungleichheitsordnung: Mehr und bessere Leistung soll einen höheren gesellschaftlichen Status begründen. Auf der anderen Seite aber ruht es auf einer fundamentalen Gleichheitsidee: Alle Gesellschaftsmitglieder sollen dieselben Chancen haben, an diesem Wettbewerb um begehrte Statuspositionen teilzuhaben. Immer wieder zeigt sich, dass beide Ziele nicht unabhängig voneinander zu erreichen sind.

\subsection{Der „Dualismus“ von Aufwand und Ergebnis}

Ein weiteres Problem in der Legitimierung einer leistungsbasierten Statushierarchie besteht darin, dass sie übergreifende Maßstäbe voraussetzt, mit denen individuelle Leistungsbeiträge miteinander verglichen und in eine Hierarchie zueinander gesetzt werden können. In einer komplexen und stark ausdifferenzierten Gesellschaft wie der unsrigen ist die Formulierung solcher Maßstäbe kein leichtes Unterfangen.

Ein erstes, wichtiges Kriterium wurde bereits genannt: Der Wert einer Leistung soll sich an den „Mühen, Kosten und Aufwendungen" orientieren, die zu ihrer Hervorbringung nötig waren (Bolte 1979, I8). Eine solche aufwandsorientierte Deutung von Leistung wird bspw. dann in Anschlag gebracht, wenn die körperliche Anstrengung und Verausgabung als Wertmaßstab herangezogen wird, die Arbeitszeit oder auch die Mühen des Qualifikationserwerbs. Aber der Aufwand kann nicht die einzige Messgröße sein. Wir müssen über Kriterien verfügen, nach denen bspw. ein anstrengendes, aber ,nutzloses" Hobby von einer gesellschaftlich produktiven Verausgabung von Arbeitskraft unterschieden werden kann. Ganz im Sinne von Durkheim bedarf es einer Vorstellung darüber, wie die Ergebnisse von individuellen Leistungsanstrengungen im Rahmen eines arbeitsteilig strukturierten Gesamtzusammenhangs sinnvoll ineinandergreifen und was in diesem Rahmen als wertvoller Beitrag gelten kann und was als sinnlose Energieverschwendung. Damit ist ein zweites wichtiges Beurteilungskriterium von Leistungen genannt, das in einer gewissen Spannung zu aufwandsorientierten Deutungen steht: das Leistungsergebnis und dessen gesellschaftliche Wertigkeit.
Claus Offe spricht hier von einem „unaustragbaren Dualismus von Leistungskriterien“ (Offe I970, 47), denn aufwands- und ergebnisorientierte Modi der Bewertung gehen keineswegs immer harmonisch zusammen. Nicht jedes Bemühen zeitigt auch die gewünschten Resultate und nicht jeder Erfolg basiert auf entsprechender individueller Anstrengung. Und in komplexen arbeitsteiligen Organisationen fällt es immer schwerer, den Beitrag Einzelner an einem gemeinsam erreichten Resultat noch individuell zuzurechnen (Voswinkel/Kocyba 2008). Tatsächlich lassen sich entlang dieses Dualismus zahlreiche historische Konflikte der Leistungsbewertung nachvollziehen: So kämpfen etwa die Gewerkschaften klassischerweise eher für eine aufwandsorientierte Leistungsbeurteilung, die Arbeitgeber beziehen sich stärker auf Ergebnisse in Form von Stückzahlen oder Markterlösen.

\subsection{Markt, Funktion, Macht - Bewertungsmodi von Leistungsergebnissen}

Mit dem Einbezug einer Ergebnisdimension in die Leistungsbewertung entstehen aber nicht nur Spannungen zu aufwandsbezogenen Kriterien. Auch auf die Frage, nach welchen Maßstäben ein jeweiliges Ergebnis als wertvoll gelten soll, kann es sehr unterschiedliche Antworten geben. Die frühen bürgerlich-liberalistischen Theoretiker (Locke, Smith, Ricardo) setzten hier vor allem auf die Kräfte des freien Marktes. Dieser sollte einen Wertindex festlegen, der sowohl die nötigen Aufwendungen (Kosten) als auch die Bedeutung eines Leistungsergebnisses widerspiegelt (Bolte 1979; Hartfiel 1977). Allerdings bringt eine solche Leistungsbewertung durch den Markt einige Probleme mit sich (Neckel/Dröge 2002). So ist Markterfolg längst nicht nur von individuellen Leistungen abhängig, sondern ebenso von der Verfügung über Kapital und Produktionsmittel sowie von günstigen Gelegenheitsstrukturen. Außerdem gibt es keinen inhärenten Zusammenhang von Aufwand und Gewinn - eher im Gegenteil: Unternehmerisches Handeln verlangt gerade nach der Minimierung des Aufwandes, um die Gewinnmarge zu steigern. Hinzu kommt, dass Märkte immer wieder Entwicklungen hervorbringen, die für das arbeitsteilige Sozialgefüge insgesamt höchst destruktiv sind - wie sich leicht am Beispiel der jüngsten Banken- und Finanzkrise nachvollziehen lässt. Will man hier regulierend eingreifen (etwa indem Bonuszahlungen begrenzt werden), so braucht es dazu jedoch eine vom reinen Markterfolg unabhängige Vorstellung darüber, welche Leistungen wünschenswert sind und welche nicht, wo also Anreize gesetzt und wo Sanktionen ausgesprochen werden sollen.

Jenseits dieser grundsätzlichen Konflikte zwischen Markt- und Leistungsprinzip haben aber auch ganz praktische Probleme mit dazu beigetragen, dass in der 
Geschichte der modernen Gesellschaft andere Kriterien der Bewertung von Leistungen ein stärkeres Gewicht erhalten haben. ${ }^{4}$ In entwickelten Industrie- sowie Dienstleistungsgesellschaften wird ein Großteil der Leistungen klassischerweise in Bereichen erbracht, die einer direkten Bewertung durch den Markt entzogen sind. Dies gilt bspw. für den öffentlichen Dienst, die Verwaltung und Politik, aber auch für die Wirtschaft selbst. Arbeit und Leistung vollziehen sich hier typischerweise im Rahmen von betrieblichen Organisationen, die nach eigenen Regeln und Gesetzmäßigkeiten funktionieren. Hier werden Leistungen nach Arbeitszeit, Qualifikationsniveau und Hierarchieposition bemessen. Mit Marktmechanismen ist dies, wenn überhaupt, nur sehr indirekt verknüpft (Menz 2009). ${ }^{5}$

Entsprechend hat sich in der sozialwissenschaftlichen Diskussion der Nachkriegszeit ein Verständnis durchgesetzt, das vor allem funktionale Gesichtspunkte in den Vordergrund rückte (Davis/Moore 1966 [1945]). Die Grundidee dieser sogenannten "funktionalen Schichtungstheorie" (ebd.) ist, dass das Leistungsprinzip auf zwei zentrale Probleme antwortet, die jede Gesellschaftsform auf die eine oder andere Weise lösen muss. Das erste ist das Problem der Allokation, also die Besetzung der verschiedenen gesellschaftlichen Positionen mit den dafür jeweils geeignetsten Personen. Das zweite Problem ist die Motivation der Personen, die ihnen übertragenen Aufgaben auch gewissenhaft zu erfüllen. Beide Probleme lassen sich aus Sicht von Davis und Moore am besten durch einen Leistungswettbewerb lösen. Dazu sei eine Statushierarchie vonnöten, die umso höhere Belohnung vorsieht (in Form von Sozialprestige, Einkommen etc.), je wichtiger eine Position für den Erhalt der Gesellschaft ist und je seltener und mühevoller zu erwerben die erforderlichen Qualifikationen seien. Dies schaffe Anreize, dass jede Person die eigene Leistungsfähigkeit bestmöglich in den Dienst der Gesellschaft stelle.

Dieser Ansatz steht unmittelbar in der Tradition des Strukturfunktionalismus von Talcott Parsons, Robert K. Merton und letztlich auch Émile Durkheim. Aber Durkheim war sehr viel sensibler dafür, dass sich die faktische Statusverteilung einer Gesellschaft einerseits und die auf das Leistungsprinzip gestützten normativen Erwartungen andererseits historisch auch auseinanderentwickeln können. Dagegen war die funktionale Schichtungstheorie optimistischer, dass die faktische

4 Im Zuge der aktuellen Ökonomisierungs- und Vermarktlichungstendenzen, die häufig mit dem Stichwort des ,Neoliberalismus' beschrieben werden, bekommt auch das klassisch-marktorientierte Verständnis von Leistung wieder neue Relevanz (Neckel/Dröge 2002).

5 Auch hier gab es in den letzten Jahren einen gewissen ,Rollback': Durch Kennziffernsysteme, Profitcenter und andere Formen von ,simulierten' Märkten innerhalb von Organisationen wird versucht, die Grenzen von Produktions- und Marktökonomie wieder durchlässiger zu machen (vgl. bspw. Wagner 2008).
Statusverteilung auch tatsächlich die funktionale Bedeutungshierarchie abbilden würde. Insbesondere diese Unterstellung hat in der Folge vielfältige Kritik auf sich gezogen (vgl. bereits Mayntz I96I).

Eine prominente Position in der deutschsprachigen Debatte nahm dabei die bereits erwähnte Studie von Claus Offe (1970) ein. Gegen die funktionale Schichtungstheorie argumentierte er, dass „extrafunktionale" Aspekte in der Leistungsbewertung tatsächlich eine große und wachsende Rolle spielen würden (vgl. ähnlich bereits Dahrendorf 1956). Wenn beispielsweise die demonstrative Loyalität zur Firma oder die Unterwürfigkeit gegenüber einem Vorgesetzten belohnt würden, so werde damit nicht Leistung, sondern Konformität zum eigentlichen gesellschaftlichen Integrationsprinzip (Offe I970, 29). Offe zufolge hat dies mit dem bereits angesprochenen Problem zu tun, dass es in einer zunehmend komplexer werdenden Industrie- und Dienstleistungsgesellschaft immer schwerer werde, klare übergreifende Kriterien zu formulieren, nach denen die funktionale Bedeutung individueller Leistungsbeiträge sinnvoll eingeschätzt und verglichen werden könne. In der Folge seien es dann vor allem die gesellschaftlichen Machtverhältnisse, die darüber entscheiden, welche Tätigkeiten oder Arbeitshaltungen als gesellschaftlich wertvoll definiert werden und welche nicht.

Offe vollzieht hier einen wichtigen Perspektivwechsel. In dem Moment, wo man die in einer Gesellschaft jeweils etablierte Werthierarchie von Leistungen nicht mehr als unmittelbaren Ausfluss funktionaler Erfordernisse oder abstrakter Marktmechanismen ansieht, öffnet dies den Blick für die sozialen Kämpfe und politischen Aushandlungsprozesse, in denen eine solche Werthierarchie erst definiert und durchgesetzt wird. Mit dieser Diagnose scheint Offe Ende der I96oer Jahre einen gewissen Zeitgeist getroffen zu haben, wie nicht nur der Erfolg seines viel diskutierten Buches belegt.

Auch außerhalb des sozialwissenschaftlichen Diskurses traten zu jener Zeit verschiedene gesellschaftliche Gruppen vermehrt in die öffentliche Arena, um ihre Vorstellungen davon, was eine gesellschaftlich wertvolle Leistung ist, politisch durchzusetzen. Die westdeutschen Gewerkschaften erlebten in den Tarifauseinandersetzungen der I960er und -70er Jahren ein "goldene[s] Zeitalter" (Schroeder 20I4, 75), die Alternativökonomie experimentierte mit Leistungsauffassungen jenseits der klassischen Tugenden des Fordismus (Dröge 2009, 93-IO3), und innerhalb der Frauenbewegung erreichte die sogenannte "Hausarbeitsdebatte“ einen ersten Höhepunkt (Paulus 2013).

Alle diese verschiedenen Ansätze und politischen Initiativen eint die grundlegende Einsicht, dass die gesellschaftliche Werthierarchie von Leistungen prinzipiell definitionsoffen und damit auch politisch verhandelbar ist. Von dieser Einsicht ausgehend können jedoch unterschiedliche ar- 
gumentative Abzweigungen eingeschlagen werden. Offe (1970) nahm seinen Befund zum Anlass, die Idee der Leistungsgesellschaft selbst fundamental zu kritisieren: Wenn die Prozesse der gesellschaftlichen Leistungsbewertung keine objektive und rationale Grundlage (mehr) hätten, dann handle es sich nur noch um eine Ideologie, die den kapitalistischen Apparat am Laufen halten solle. Andere Ansätze, wie bspw. die Anerkennungstheorie von Axel Honneth (1994), gehen hier weniger weit und richten den Blick stärker auf die sozialen Aushandlungsprozesse, die hinter der historischen Gewordenheit bestimmter Leistungsverständnisse stehen.

Wie der heutige Stand dieser Deutungs- und Auslegungskämpfe um das Leistungsprinzip ist, soll jetzt anhand eigener Forschungen exemplarisch nachgezeichnet werden.

\section{Empirische Deutungskonflikte um ,Leistung}

Welche normativen Vorstellungen und Erwartungen zum Thema Leistung heute in der deutschen Gegenwartsgesellschaft vorzufinden sind, haben Sighard Neckel, Irene Somm und ich vor einiger Zeit in einem größeren Forschungsprojekt am Institut für Sozialforschung in Frankfurt am Main untersucht, das von der Deutschen Forschungsgemeinschaft DFG finanziert wurde (Dröge/Somm 2005; Neckel et al. 2004; Neckel et al. 2005). Dazu wurden I5 rund zweistündige Gruppendiskussionen mit über 80 Teilnehmenden aus ganz unterschiedlichen sozialen Milieus und Tätigkeitsfeldern durchgeführt. Die Diskussionen gliederten sich in einen ersten Teil, in dem die Diskutanten anhand eines konkreten „Szenarios“ über Rechtfertigungsgründe gesellschaftlicher Statuszuweisung debattierten sowie einen zweiten, der stärker die eigenen Erfahrungen mit Leistung und Leistungsbewertung zum Gegenstand hatte (zum empirischen Vorgehen vgl. ausführlich Dröge et al. 2006). Daraus lässt sich sowohl rekonstruieren, welche normativen Maßstäbe die Befragten an die gesellschaftliche Statusordnung insgesamt anlegen, als auch, wie sie sich selbst darin verorten.

Die Ergebnisse wurden idealtypisch zu fünf allgemeinen „Bewertungsrahmen“ von Leistung verdichtet. Wir sprechen hier in Anlehnung an Erving Goffman (1976) von „Rahmen“, weil diese Konzepte fünf empirisch-typologisch unterscheidbare Hinsichten darstellen, in denen sich ,Leistung ' heute inhaltlich ausdeuten und beurteilen lässt. Diese Bewertungsrahmen haben also einen sehr viel höheren Konkretionsgrad als die oben ideengeschichtlich herausgearbeiteten allgemeinen Kernelemente des Leistungsprinzips. Sie sind aber gleichwohl idealtypisch verdichtet und kommen empirisch zumeist nicht in Reinform, sondern eher in variablen Mischungsverhältnissen vor.
Ich kann die Ergebnisse dieser Forschung hier nicht in aller Breite ausführen (vgl. dazu ausführlicher Neckel et al. 2005; Dröge/Somm 2005; Neckel et al. 2004; Dröge/Neckel 20I0; Dröge 2007), sondern werde nur zwei Bewertungsrahmen herausgreifen, deren Vergleich für die Frage der Sozialintegration besonders interessant ist. Dabei kommt es mir insbesondere darauf an zu zeigen, wie hier jeweils mit den oben herausgearbeiteten internen Konflikten des Leistungsprinzips umgegangen wird, welche Integrations- und Anerkennungsversprechen impliziert sind und wo die ausgrenzenden und missachtenden Potentiale liegen.

\subsection{Leistung als Arbeit}

Leistung als ,Arbeit' zu beschreiben, wirkt fast schon tautologisch - so eng ist der Leistungsbegriff mit den sich wandelnden gesellschaftlichen Arbeitsverständnissen verknüpft. Historisch war die Neubewertung der Arbeit durch den Protestantismus und insbesondere den Calvinismus eine wichtige Voraussetzung für die Herausbildung der Idee der Leistungsgesellschaft (Weber 1988). Entsprechend zentral ist auch heute noch die Vorstellung, dass ein gesellschaftlicher Statusgewinn dann leistungsgerecht ist, wenn er auf eigene, harte Arbeit zurückgeführt werden kann. Dieses Deutungsmuster war auch in unseren Gruppendiskussionen immer wieder präsent. Aber es wurde ebenso sichtbar, dass dieser Bezug auf Arbeit als zentrale Referenzgröße für Leistung heute mit vielfältigen Problemen behaftet ist.

Ein Facharbeiter eines großen deutschen Chemiewerkes beispielweise äußert sich in der Gruppendiskussion etwas verzweifelt: „Ich seh' nicht, was ich leiste. Bei uns ist die ganze Produktion in den Rohren, die fliegt durch die Rohre." Greifbar und sinnlich erfahrbar wird die eigene Leistung für ihn eher im Privaten, wo er seinen Stolz daraus zieht, sein Haus mit eigenen Händen errichtet zu haben. In seinem Beruf hingegen besteht seine Tätigkeit hauptsächlich darin, in einer Messwarte die automatisierte Produktion auf Computermonitoren zu überwachen. Es fällt ihm offensichtlich schwer, diese Tätigkeit in derselben Weise als individuelle Leistung anzusehen, denn es fehlt ihr die sinnlich-unmittelbare Dimension der Aktivität auf dem Bau, die körperliche Verausgabung, der direkte Kontakt mit dem Material, das durch die eigenen Hände zu einem Werk geformt wird, kurz: Es fehlt das, was aus seiner Sicht ,Arbeit' im eigentlichen Sinne ausmacht.

Hier schlägt sich eine Problematik nieder, die schon Claus Offe (1970) herausgestellt hat: Wenn körperliche Tätigkeiten vermehrt durch Maschinen ersetzt (oder ins Ausland verlagert) werden und die ,Wissensarbeiten in Kontrolle, Verwaltung und Dienstleistung einen immer breiteren Raum einnehmen, dann wird die individuelle Leistung zunehmend abstrakter, unsichtbarer und schwerer 
fassbar. Insbesondere die Aufwandsdimension von Leistung wird problematisch: Individuelle Anstrengung ist nirgends sinnfälliger als in der körperlichen Arbeit.

Für die Arbeiterschaft war das körperliche Moment ihrer Tätigkeit immer die Quelle eines eigenen Leistungsstolzes, der sich auch in unseren Gruppendiskussionen vereinzelt in ironischen Bemerkungen über die „Köfferchenträger" in der Verwaltung und im Management niederschlägt, die vor allem „Sitzfleisch“ ausbilden, aber sonst kaum erkennbare Arbeitsleistungen erbringen würden. Allerdings zeigt das Beispiel des oben zitierten Chemiearbeiters, dass auch innerhalb der Arbeiterschaft viele Tätigkeiten inzwischen weit von dem Idealbild körperlicher Leistung entfernt sind. Hinzu kommt das Problem, dass körperliche Arbeit heute häufig die Form von "Normalisierungsarbeit" (Voswinkel/Korzekwa 2005) annimmt oder auf andere Weise auf eine verborgene „Hinterbühne“ (Goffman) verbannt wird. Normalisierungsarbeit ist dadurch gekennzeichnet, dass es vor allem um die Aufrechterhaltung eines spezifischen Status quo und die Beseitigung von Fehlern und Störungen geht. Erreicht diese Arbeit ihr Ziel, so bleibt sie weitgehend unsichtbar, die Abläufe sind ,normal' und störungsfrei.

Ein Beispiel aus unseren Gruppendiskussionen ist die Tätigkeit der Beschäftigten in einem Supermarkt, deren Aufgabe wesentlich darin besteht, die Regale und Tiefkühltruhen immer gefüllt zu halten und generell für einen reibungslosen Ablauf der Prozesse im Ladenlokal zu sorgen. Diese Arbeit ist körperlich anstrengend und die Beschäftigten ziehen einen wichtigen Teil ihres Leistungsstolzes aus der Schnelligkeit und Effizienz, mit der sie ihre Tätigkeiten verrichten. Aber diese Aktivitäten sollen im normalen Betrieb möglichst wenig auffallen. Beachtung finden sie vor allem dann, wenn sie misslingen, wenn fehlende Waren oder gestörte Abläufe die Aufmerksamkeit von KundInnen und Vorgesetzten auf sich ziehen. Hier zeigt sich ein Anerkennungsproblem: Erfahrungen positiver Wertschätzung sind selten, negative Beachtung bei Fehlern oder Unzulänglichkeiten dagegen sehr viel wahrscheinlicher.

Generell haben wir einen starken Bezug auf Arbeit als zentralen Maßstab der Leistungsbewertung in unserem Sample fast ausschließlich bei niedrigqualifizierten Gruppen gefunden. Ein arbeitszentriertes Leistungsverständnis verspricht heute vor allem noch jenen Personen gewisse Integrationschancen, denen Bildungstitel fehlen oder die aus anderen Gründen keinen Zugang zu qualifizierteren Tätigkeiten bekommen. Allerdings ist dieses Integrationsversprechen in mehrerer Hinsicht brüchig: Wie gezeigt, bietet die Arbeitswelt der westlichen Gesellschaften heute immer weniger praktische Anwendungsbedingungen für ein solches Leistungsverständnis, hinzu kommen die spezifischen Sichtbarkeits- und Anerkennungsprobleme der "Normalisierungsarbeit".
Aber auch aus einen dritten Grund ist die praktische Integrationsfähigkeit des arbeitszentrierten Leistungsverständnisses zweifelhaft. Keine der Gruppen, die im Blick auf die Beurteilung der eigenen Leistung stark auf den Faktor Arbeit abstellten, war in der Lage, diesen Bewertungsmaßstab auch über die eigene Soziallage hinaus zu verallgemeinern und auf höhere Statusgruppen anzuwenden. Offenbar sind sie selbst der Ansicht, einem inzwischen partikularen und weitgehend überholten Leistungsverständnis anzuhängen, das nur noch in wenigen Inselbereichen der heutigen Arbeitswelt sinnvolle Anwendungsbedingungen vorfindet. ${ }^{6}$ Damit fehlt ihnen jedoch auch ein Vergleichsmaßstab, um die eigenen Leistungen plausibel zu denen anderer gesellschaftlicher Gruppen in ein Verhältnis setzen zu können. Mit Durkheim kann man sagen, dass sie kaum in der Lage sind, sich selbst als Teil eines durch organische Solidarität verbundenen größeren sozialen Ganzen zu begreifen. Stattdessen zerfällt die Gesellschaft aus ihrer Sicht in eine relativ unverbundene Dichotomie von ,oben "und, unten', in zwei Welten, in denen Anerkennung für Leistung nach jeweils ganz eigenen Kriterien verteilt wird (Neckel et al. 2005; vgl. auch bereits Popitz et al. 1957). Was bleibt, ist ein diffuses Unrechtsempfinden sowie eine ansonsten eher fatalistische Sicht auf die Sozialwelt und die eigene Position darin. Als gelungene soziale Integration kann man dies nur schwerlich bezeichnen.

\subsection{Die Leistung der Person}

Nachdem mit dem Bewertungsrahmen ,Arbeit' ein klassisches, heute brüchiges Leistungsverständnis in den Blick genommen wurde, möchte ich mich jetzt einem jüngeren Deutungsmuster zuwenden. Im Kontext des Bewertungsrahmens ,Person" wird ein Handeln dann als belohnenswerte Leistung anerkannt, wenn sich in ihm spezifische Eigenheiten, Fähigkeiten und Potenziale einer Person in authentischer Weise äußern. Selbstverantwortung, Selbstmotivation und Selbstentfaltung sind wichtige Stichworte in diesem Zusammenhang (vgl. ausführlich Dröge 2007). Hier schlägt sich ein sozialer und ökonomischer Wandel nieder, der generell unter dem Stichwort „Subjektivierung der Arbeit“ (Baethge I99I; Moldaschl/ Voß 2002) verhandelt wird.

Seine historischen Wurzeln hat diese Entwicklung im Wertewandel der 1960er und -70er Jahre (Bell I99I [1976]), der den sogenannten „postmaterialistischen“ Orientierungen an Selbstverwirklichung und einem sinnerfüllten (Arbeits-)Leben in verschiedenen gesellschaftlichen Milieus zu wachsender Bedeutung verhalf. Mittlerweile sind diese zunächst eher kritischen Ori-

6 Aktuelle empirische Befunde zeigen, dass ein solches Bewusstsein der Partikularität der eigenen normativen Ansprüche an Arbeit sich offenbar bis weit in die gesellschaftliche Mitte hinein auszubreiten beginnt (Hürtgen/Voswinkel 20I4; Dörre et al. 2OI3). 
entierungen in die kapitalistischen Arbeitsprozesse integriert worden (Boltanski/Chiapello 2003). Insofern bezeichnet der Begriff der "Subjektivierung der Arbeit“ heute zwei miteinander verschränkte Entwicklungsprozesse (Kleemann et al. 2002): Zum einen ein Wandel des Arbeitsbewusstseins: Die Beschäftigten sehen die Erwerbsarbeit heute stärker als Feld der individuellen Selbstverwirklichung, in das sie sich mit ihren je eigenen Fähigkeiten, Talenten und Neigungen einbringen möchten („normative Subjektivierung der Arbeit“, Baethge 199I). Zum zweiten wird aber auch ein Wandel der betrieblichen Anforderungen diagnostiziert. Moderne Managementkonzepte und Organisationsmodelle fordern Selbststeuerung und Selbstverantwortung, Kreativität und persönliches Engagement (Kocyba 2000). Wir können aus unseren eigenen Forschungen diese beiden Entwicklungstendenzen noch um eine dritte ergänzen: Nicht nur im Arbeitsbewusstsein und im betrieblichen Umfeld, sondern ebenso im Blick auf die Rechtfertigung der gesellschaftlichen Statusordnung insgesamt gewinnen diese Kriterien an Bedeutung.

In einer ganzen Reihe unserer Gruppendiskussionen war Authentizität ein zentrales Kriterium der Leistungsbewertung. Wertgeschätzt wird, wenn eine Person sich mit ihren je individuellen Fähigkeiten und Eigenheiten selbstgesteuert in die Tätigkeit einbringt und an persönlichen Zielen auch gegen äußere Widerstände festhält. Populäre Vorbildfiguren sind hier bspw. der AppleMitbegründer Steve Jobs, der - so die mediale Erzählung - seinen Erfolg vor allem dieser Kombination aus kreativem Eigensinn und Durchhaltewillen verdankt. Auf größte Ablehnung stößt in den betreffenden Gruppen dagegen die Belohnung von Angepasstheit. Mit Tzvetan Todorov (1996, 98) kann man sagen: Hier wird „Distinktionsanerkennung“ ausgesprochen und „Konformitätsanerkennung" delegitimiert.

Aus der Sicht unserer DiskutantInnen dient die Selbstentfaltung jedes/r Einzelnen in der Summe auch dem Gemeinwohl, weil nur so die ganze Breite der in einer Gesellschaft vorhandenen Talente und Begabungen ausgeschöpft und für das Gemeinwesen insgesamt nutzbar gemacht werden kann. Insofern ist die individuelle Selbstverwirklichung in ein durch „organische“ Solidarität verbundenes Netz sozialer Reziprozitätsbeziehungen eingebettet und kann somit auch als Leistung interpretiert werden, die einen Anspruch auf gesellschaftliche Gegenleistung begründet.

Außerdem führt das Selbstverwirklichungsideal auch keineswegs zu einem leistungsfeindlichen Hedonismus, wie früher teils befürchtet (Bell I99I; NoelleNeumann 1978). Deutlich wird dies beispielsweise in einer Gruppendiskussion mit hochqualifizierten Kleinunternehmerinnen aus dem Kultur- und Beratungsbereich, die sämtlich unter prekären ökonomischen Bedingungen arbeiten. Teilweise müssen sie für Aufträge sehr viel mehr Arbeit investieren, als angesichts der vereinbarten Bezahlung eigentlich gerechtfertigt wäre. Aber anders als früher im Angestelltenverhältnis, wo sie sich „ausgelaugt und ausgewrungen“ fühlten, bekommt dieser Aufwand nun eine andere Bedeutung. Er wird dadurch geadelt, dass er im Dienste des eigenen Unternehmens erbracht wird, das immer auch für ein individuelles Selbstverwirklichungsprojekt steht: „Das ist schon etwas, was anders ist, wenn man das für sich selber macht, ... auch wenn man aus dem letzten Loch pfeift, weil's unglaublich viel ist". Authentizität hat in diesem Sinne immer einen Aktivitäts- bzw. Aufwandsbezug; sie muss aktiv angestrebt, durchgesetzt und nötigenfalls unter Opfern verteidigt werden (vgl. bereits Brooks 200I, 156). Insofern ist sie auch als Leistung thematisierbar, die gesellschaftliche Gegenleistung rechtfertigt.

Allerdings sind diese Beobachtungen in einer wichtigen Hinsicht einzuschränken. Denn keiner der Bewertungsrahmen ist in unserem Material so ungleich verteilt wie jener der Person. Die Scheidungslinie verläuft vor allem entlang von Milieu- und Bildungsunterschieden. In allen hochqualifizierten Gruppen unseres Samples finden sich Bezüge auf diesen Rahmen; in niedrigqualifizierten Gruppen hingegen ist dies eine überaus seltene Ausnahme. Andere Untersuchungen zur „Subjektivierung der Arbeit" bestätigen diese Ungleichverteilung, behandeln sie jedoch eher am Rande (Baethge 199I, I2; Kleemann et al. 2002, 83). Für die Frage nach der Struktur der gesellschaftlichen Anerkennungsordnung ist dieser Befund jedoch von zentraler Bedeutung. Die Orientierung auf Selbstverwirklichung in einer subjektivierten Arbeitswelt ist offenbar stark in höheren gesellschaftlichen Schichten konzentriert, wird jedoch von diesen souverän zu einem generellen Maßstab der Leistungsbewertung verallgemeinert. Dabei können sie sich in einem Zeitgeist abgesichert sehen, der das Selbstverwirklichungsideal $\mathrm{zu}$ einem zentralen gesellschaftlichen Wert erhebt. Niedrigqualifizierte Gruppen dagegen beziehen ihren Leistungsstolz eher aus Elementen des Bewertungsrahmens Arbeit, können dies aber kaum über die eigene Gruppe hinaus verallgemeinern. Einmal mehr scheint also die dominante gesellschaftliche Auslegung des Leistungsprinzips dem habituellen Vorbild privilegierterer Schichten zu folgen, das zu einem generellen Prinzip öffentlicher Anerkennung verallgemeinert wird. Dies benachteiligt jene sozialen Gruppen, die sich in ihrem Leistungsverständnis an anderen normativen Bezügen orientieren - sei es aufgrund milieuspezifischer kultureller Differenzen, oder sei es schlicht aus Ermangelung der Möglichkeit, die eigene Arbeit plausibel in den Kategorien des Selbstverwirklichungsideals zu interpretieren.

Aber noch in einer anderen Hinsicht tangieren die Subjektivierung von Arbeit und Leistung den normativen Kernbestand des Leistungsprinzips. Denn die For- 
derung nach Chancengleichheit hat in einer Bewertungslogik, die sich an der Authentizität der Person und dem Ideal der individuellen Selbstverwirklichung orientiert, kaum noch einen Platz. Dies zeigt sich exemplarisch in der folgenden Äußerung einer Bankmitarbeiterin:

Ich denke, man hat immer irgendwas. Sei es, dass man sein Äußeres hat, sei es, dass man seine Intelligenz hat, seine Fähigkeiten, und sei es, dass man eine gewisse Erziehung hat vom Elternhaus, oder sei es, dass man von Eltern oder von den Vorfahren irgendwas ererbt, ob jetzt genetisch oder materiell oder sonst wie. Also ich finde, man hat immer irgendetwas und jeder hat halt etwas. ... Erfolg bedeutet für mich, wenn man aus diesem Etwas, was auch immer das sein mag, ja, etwas schafft.

In dieser Äußerung verbinden sich implizit zwei Einwände gegen die normative Forderung nach Chancengleichheit, die für Argumentationen im Kontext des Bewertungsrahmens Person generell typisch sind. Zum ersten gilt die Forderung nach Chancengleichheit schon deshalb als problematisch, weil jede Person einzigartig ist und eine Schaffung gleicher Startbedingungen daher, so die Unterstellung, einer Entindividualisierung gleichkommen würde, die weder praktikabel noch wünschenswert ist. Nun wissen wir allerdings spätestens seit den Analysen von Pierre Bourdieu, dass die so verstandene ,Einzigartigkeit' einer Person immer auch maßgeblich durch ihre soziale Herkunft mit bestimmt ist (Bourdieu I987), und wir wissen, dass sich auf diesem Weg soziale Ungleichheit über die Generationen hinweg reproduziert. Bei Bourdieu allerdings musste sich die soziale Platzierungswirkung des kulturellen Kapitals noch „im Verborgenen" (Bourdieu 1983, I87) vollziehen, um die „Illusion der Chancengleichheit" (Bourdieu/Passeron 197I) nicht zu gefährden. Heute dagegen können wir beobachten, dass die Vorteile einer privilegierten Herkunft häufig ganz der Individualität des Einzelnen und dem authentischen Wesenskern zugeschlagen werden. Dann können sie unter dem Dach der Selbstverwirklichungsidee ganz offen zu einer legitimen Ressource im gesellschaftlichen Statuswettbewerb werden - „Diversity" ist das populäre Stichwort dazu. Die Entproblematisierung herkunftsbedingter Vorteile kann, wie das obige Zitat in ungewöhnlicher Deutlichkeit zeigt, so weit gehen, dass selbst der Transfer von ökonomischem Kapital zwischen den Generationen keinen Anlass mehr bietet, die Frage der Chancengleichheit bei der Leistungsbewertung zu berücksichtigen. Paradoxerweise kehren hier eigentlich ständische, weil herkunftsbegründete Bewertungskriterien in Gestalt eines höchst modernen, ganz dem Individualismus verschriebenen Argumentationsmusters zurück.
Diese weit reichende Entproblematisierung sozial ungleicher Startbedingungen begründet sich zusätzlich aus einem zweiten Argument, das ebenfalls in dem obigen Zitat enthalten ist und das als gleichermaßen typisch für den Bewertungsrahmen Person gelten kann. Dieses unterstellt, dass die Menschen in ihrer Einzigartigkeit auch wieder alle gleich sind. "Jeder hat halt etwas" und jeder kann sich selbst verwirklichen - wenn nicht im Beruf, dann im Privatleben, im ehrenamtlichen oder politischen Engagement, etc. Selbstverwirklichung gilt nicht als ein knappes Gut, weil sie begrenzter Ressourcen wie Macht, Geld, Titel oder Stelle nicht notwendig bedarf. Daher scheint Chancengleichheit auf einer abstrakteren Ebene per se gegeben. Weitgehend ausgeblendet wird in dieser Sichtweise jedoch, dass der individuelle Wunsch zur Selbstentfaltung auf sehr unterschiedliche gesellschaftliche Realisierungschancen treffen kann. Nicht jede berufliche Tätigkeit lässt sich plausibel als authentischer Ausdruck des eigenen Selbst interpretieren. Und auch sonst ist Selbstverwirklichung an sozial ungleich verteilte Voraussetzungen geknüpft - allen voran ein gewisses $\mathrm{Ma}$ an ökonomischer Sicherheit und sozialer Integration, ohne die die Autonomisierung des Einzelnen in einen "negativen Individualismus" (Castel 2000, 4OI) zu kippen droht, der kaum noch Wahlmöglichkeiten und Selbstentfaltungschancen bietet.

Insgesamt zeigen diese Beobachtungen, dass die „Subjektivierung von Arbeit“ den gesellschaftlich höher stehenden Sozialgruppen neue Möglichkeiten zur Legitimierung und Absicherung ihrer eigenen Statusposition gegeben hat. Aber die Anerkennung subjektivierter Arbeit kennt auch eigenen Schwierigkeiten, die sich vor allem im alltäglichen Ringen um die Wertschätzung der eigenen Leistung durch Vorgesetzte, KollegInnen, KundInnen, etc. zeigen. Hier kollidieren die Anerkennungserwartungen mit der Idee von Authentizität, die dem Bewertungsrahmen Person zugrunde liegt. Sie fordert, dass der oder die Einzelne ganz intrinsischen Motiven folgt. Wer offensiv Anerkennung einfordert, läuft daher Gefahr, als „außengeleiteter Charakter" (Riesman) zu erscheinen, der sich in der eigenen Selbstverwirklichung von der Meinung Anderer abhängig macht. Eine Teilnehmerin unserer Studie, die bildende Kunst studiert hat und heute ein eigenes kleines Marketingbüro betreibt, bringt dieses Problem wie folgt auf den Punkt:

Ich bin eigentlich, so, glaube ich, ich bin eine ganz pfiffige Marketing-Frau. Dadurch, dass ich aber dieses künstlerische Denken im Kopf habe, dass mein Auftrag dann erfüllt ist, wenn alle sagen: Oh, toll, Frau X, zehntausend Euro sind für Sie, ne? ... Das ist bei mir 'ne absolute Schwachstelle. Das ist so'n Hang zur Selbstausbeutung, eigentlich. 
Was hier als Spezifikum des „künstlerischen Denkens“ klassifiziert wird, ist tatsächlich das generelle Problem eines Reziprozitätskonzeptes, das sich mit der Authentizitätsidee zu verbinden sucht (zur Verallgemeinerung eines ,künstlerischen' Arbeitsethos vgl. auch Boltanski/ Chiapello 2003). Man kann Anerkennung erhoffen, aber wenn diese ausbleibt, ist sie kaum moralisch einklagbar. Vor allem kann man sich schwer auf gesellschaftliche Normen und Standards berufen - etwa die Zahlung eines zuvor festgesetzten Preises für die pflichtgemäße Erfüllung eines Auftrages. Die Anerkennung soll ja gerade das Besondere der jeweiligen Person und ihrer ,authentischen' Leistung zum Gegenstand haben: „Oh, toll, Frau X, zehntausend Euro sind für Sie“.

Stephan Voswinkel (2002) beschreibt diesen Anerkennungsmodus treffend als "Bewunderung", den er vom Modus der „Würdigung" unterscheidet. Würdigung honoriert die Pflichterfüllung und den Beitrag des Einzelnen zu einem kollektiven Leistungshandeln und ist eher für das oben dargestellte arbeitszentrierte Leistungsverständnis typisch. Bewunderung hingegen richtet sich auf das Besondere, Herausragende, Einzigartige oder Überlegene. Sie kennt keine Verpflichtung zur Reziprozität und kann daher auch nicht moralisch eingefordert werden. Im Ergebnis müssen sich die Individuen "fehlende Anerkennung ... selbst zurechnen, eben weil sie keinen Anlass zur Bewunderung geboten haben" (Voswinkel 2002, 84).

Man sieht, dass auch ein auf die ,Person' und deren authentische Selbstverwirklichung in der Arbeit ausgerichtetes Leistungsverständnis eine durchaus ambivalente Form sozialer Integration begründet. Einerseits bietet sich hier - im besten Falle - tatsächlich die Möglichkeit, als einzigartiges Individuum für den je besonderen eigenen Beitrag zu einem arbeitsteiligen Gemeinwesen soziale Anerkennung zu erhalten. Andererseits aber macht man sich dadurch von einer spezifischen Form der Anerkennung abhängig, die, wenn sie ausbleibt, nur schwer normativ einzufordern ist. Weitere Schwierigkeiten ließen sich ergänzen, etwa jene, die daraus entstehen, dass Selbstverwirklichung und Authentizität heute nicht nur individuelle Arbeitsansprüche, sondern auch organisationale Erwartungen darstellen - was in paradoxale Formen einer Art ,fremdbestimmter Selbstbestimmung münden kann (Honneth 2002).

\section{Fazit}

Die vorliegende Analyse hat sich aus zwei grundsätzlichen Richtungen der Frage nach den integrativen Potentialen des Leistungsprinzips genähert. Zunächst wurde mit Axel Honneth argumentiert, dass Leistung ein zentrales Anerkennungsprinzip der modernen Gesellschaft darstellt (Abschnitt I). Die sozial integrative Wirkung dieses Prinzips beruht darauf, dass das Individuum sich durch die Anerkennung seiner Leistungen als ,wertvolles' Mitglied eines Gemeinwesens erfahren kann, dessen Beiträge für den arbeitsteiligen Gesamtzusammenhang von Bedeutung sind. Entsprechend groß ist das Desintegrationspotential, wenn Individuen oder Gruppen sehen, dass ihre Leistungsbeiträge missachtet oder ignoriert werden. Weiter wurde argumentiert, dass das Leistungsprinzip eine Reihe von wichtigen Kernelementen umfasst, die jede gesellschaftliche Auslegung dieses Prinzips mehr oder weniger in Betracht ziehen muss (Abschnitt 2). Diese Kernelemente wurden in Form einer ideengeschichtlichen Rekonstruktion herausgearbeitet. Sie beinhalten die grundlegende Vorstellung einer Reziprozität zwischen Individuum und Gesellschaft, die wiederum auf dem Selbstverständnis einer arbeitsteiligen Sozialordnung aufruht, in der die oder der Einzelne von den Leistungsbeiträgen Anderer abhängig ist. Hinzu kommen das Prinzip der Chancengleichheit, aufwands- und ergebnisbezogene Leistungskriterien sowie verschiedene Modi der Leistungsbewertung (etwa Markt, funktionale Bedeutsamkeit, etc.).

Dabei wurde jedoch ebenfalls sichtbar, dass schon auf dieser allgemeinen Ebene vielfältige Konflikte angelegt sind: der "unaustragbare Dualismus" (Offe 1970, 47) zwischen Aufwand und Ergebnis; das spannungsreiche Verhältnis zwischen Gleichheitsidee und Ungleichheitsordnung, das sich in Konflikten um die Verwirklichung von Chancengleichheit niederschlägt; die Schwierigkeiten, in einer komplexen arbeitsteiligen Gesellschaft sinnvolle Vergleichsmaßstäbe zu formulieren, mit denen sich der individuelle Beitrag Einzelner an einem kollektiven Leistungsergebnis fair abschätzen lässt; etc.

Vor diesem Hintergrund wird verständlich, warum ,Leistung' in der Moderne immer auch ein umkämpfter Begriff war und ist. Ich habe versucht zu zeigen, dass die internen Spannungen des Leistungsprinzips grundsätzlicher Natur sind und nicht einfach einer gewissen Unschärfe des Konzepts geschuldet, die durch eine kluge und vernünftige politische Ausdeutung leicht zu beseitigen wäre. Der Dualismus von Aufwand und Ergebnis ist nicht auflösbar, sondern „unaustragbar", wie Offe formuliert. Die Spannungen zwischen Gleichheitsideal und Ungleichheitsordnung lassen sich vielleicht für einen spezifischen historischen Moment einigermaßen zufriedenstellend ausbalancieren, aber immer nur in Gestalt eines Kompromisses, der auch Verliererinnen und Verlierer produziert. So ist die Eingangsthese zu verstehen, dass jede auf Leistung gegründete Form der gesellschaftlichen Statusorganisation sowohl anerkennende als aus missachtende Aspekte enthält und damit sowohl integrative als auch desintegrierende Wirkungen entfaltet. Dies wiederum hält die Dynamik der gesellschaftlichen Kämpfe um Anerkennung beständig in Gang. 
Allerdings sind es nicht nur die internen Ambivalenzen, Spannungen und Konflikte des Leistungsprinzips, die in solchen gesellschaftlichen Deutungskämpfen ihren Ausdruck finden. Hinzu kommt, dass auch die genannten allgemeinen Kriterien für Leistung selbst einer inhaltlichen Auslegungen und Adaption bedürfen, um in einem konkreten historischen Kontext auf eine konkrete Statusordnung angewendet werden zu können. Der zweite Hauptteil des Beitrages (Abschnitt 3) hat dies unter Rückgriff auf eigene Forschungen empirisch beispielhaft gezeigt. Dabei wurde ein klassisches, arbeitsbezogenes Leistungsverständnis einem neueren gegenübergestellt, das sich vor allem auf Selbstverwirklichung und den authentischen Ausdruck der Person im Leistungshandeln stützt.

In diesen empirischen Leistungsverständnissen tauchen die oben herausgearbeiteten grundlegenden Spannungen und Ambivalenzen in modifizierter Form wieder auf. Das Prinzip der Chancengleichheit beispielweise erfährt in beiden Sichtweisen eine sehr unterschiedliche Auslegung. Für ein arbeitszentriertes Leistungsverständnis ist Chancengleichheit zentral, es begreift sich als in hohem Maße sozial integrativ: Tüchtigkeit, Einsatzbereitschaft und ,harte Arbeit' kann jede und jeder erbringen, so die Auffassung, unabhängig von Bildungstiteln oder sozialer Herkunft, ja, vielleicht sogar unabhängig von körperlichen Handicaps. Probleme der Chancengleichheit ergeben sich eher aus externen Einflüssen - vor allem aus dem Wandel der Arbeitswelt, die einem solchen Leistungsverständnis immer weniger praktische Realisierungschancen bietet. Das ,personenzentrierte' Leistungsverständnis hingegen blendet die Frage der Chancengleichheit weitgehend aus. Jede und jeder ist anders, so die Auffassung, und wird daher auch eigene Wege der Selbstverwirklichung beschreiten. Die sozial ungleich verteilten Chancen werden dethematisiert und im Idealbild einer bunten, durch Vielfalt und "Diversity" gekennzeichneten Gesellschaft aufgelöst.

Eine weitere Einsicht aus der empirischen Rekonstruktion ist, dass die historischen Veränderungen der konkreten gesellschaftlichen Leistungsverständnisse häufig mit sozialen und kulturellen Transformationsprozessen verknüpft sind, die nicht aus den leistungsbezogenen Anerkennungskämpfen selbst hervorgehen. So hat die heute eher marginale Rolle des arbeitszentrierten Leistungsverständnisses vor allem mit ökonomischen und technologischen Veränderungen zu tun. Der Aufstieg des personenzentrierten Leistungsverständnisses wiederum wäre undenkbar ohne die kulturellen Wandlungsprozesse im sozialen Wertehaushalt, die dem Selbstverwirklichungsideal einen neuen und wichtigen gesellschaftlichen Stellenwert gegeben haben. Diese externen Einflüsse sind eine zweite Quelle, die die Dynamik der Anerkennungskämpfe und der Transformation der gesellschaftlichen Leistungsverständnisse zusätzlich antreibt.

Insgesamt betrachtet kann also die Eingangsfrage, inwiefern das Leistungsprinzip sozial integrierend wirkt oder eher spaltet und trennt, mit einem deutlichen ,sowohl als auch beantwortet werden. Ohne Zweifel stellt Leistung in modernen Gesellschaften einen zentralen und unhintergehbaren Modus der Sozialintegration dar. Aber jede Anwendung dieses Prinzips in der sozialen Praxis produziert blinde Flecken, hebt bestimmte Leistungsbeiträge hervor und marginalisiert andere bzw. macht sie sozial unsichtbar. Und gerade weil dieses Prinzip für die Sozialintegration der modernen Gesellschaft so unhintergehbar ist, sind die desintegrierenden und potentiell anomischen Folgen dieser Invisibilisierung gravierend und sollten weder der sozialwissenschaftlichen noch der politischen Aufmerksamkeit entgehen.

\section{Literatur}

Baethge, Martin (199I). Arbeit, Vergesellschaftung, Identität - Zur zunehmenden normativen Subjektivierung der Arbeit, in: Soziale Welt, Vol. 42(I), 6-19.

Bell, Daniel (199I). Die kulturellen Widersprüche des Kapitalismus, Frankfurt am Main/New York.

Boltanski, Luc/Ève Chiapello (2003). Der neue Geist des Kapitalismus, Konstanz.

Bolte, Karl Martin (1979). Leistung und Leistungsprinzip. Zur Konzeption, Wirklichkeit und Möglichkeit eines gesellschaftlichen Gestaltungsprinzips. Ein Beitrag zur Sozialkunde der Bundesrepublik Deutschland, Opladen.

Bourdieu, Pierre (1983). Ökonomisches Kapital, kulturelles Kapital, soziales Kapital, in: Reinhard Kreckel (Hg.): Soziale Ungleichheiten (Sonderband 2 der Sozialen Welt), Göttingen, I83-I98.

Bourdieu, Pierre (1987). Die feinen Unterschiede. Kritik der gesellschaftlichen Urteilskraft, Frankfurt am Main.

Bourdieu, Pierre/Jean-Claude Passeron (197I). Die Illusion der Chancengleichheit. Untersuchungen zur Soziologie des Bildungswesens am Beispiel Frankreich, Stuttgart.

Brooks, David (200I). Die Bobos. Der Lebensstil der neuen Elite, München.

Castel, Robert (2000). Die Metamorphosen der sozialen Frage. Eine Chronik der Lohnarbeit, Konstanz.

Dahrendorf, Ralf (1956). Industrielle Fertigkeiten und soziale Schichtung, in: Kölner Zeitschrift für Soziologie und Sozialpsychologie, Vol. 8, 540-568.

Davis, Kingsley/Wilbert E. Moore (1966). Some Principles of Stratification, in: Reinhard Bendix/Seymour Martin Lipset (Hg.): Class, Status, and Power. Social 
Stratification in Comparative Perspective (Second Edition), New York, 47-53.

Dörre, Klaus/Anja Hänel/Ingo Matuschek (Hg.) (2013). Das Gesellschaftsbild der LohnarbeiterInnen. Soziologische Untersuchungen in ost- und westdeutschen Industriebetrieben, Hamburg.

Dröge, Kai (2007). „Jetzt lob' mich doch endlich mal!“ Subjektivierte Arbeit und die Fallstricke ihrer Anerkennung, in: Anette Henninger/Christine Wimbauer/ Markus Gottwald (Hg.): Die Gesellschaft als ,institutionalisierte Anerkennungsordnung' - Anerkennung und Ungleichheit in Paaren, Arbeitsorganisationen und Sozialstaat, Opladen, 97-II7.

Dröge, Kai (2009). Der Unternehmer. Aufstieg und Fall eines gesellschaftlichen Leitbildes. Dissertation, Justus Liebig Universität Gießen.

Dröge, Kai/Sighard Neckel (2010). Leistungsbilanzen. Ein Deutungsmuster verflüchtigt sich - und bleibt umkämpft, in: polar(8), 7-I3.

Dröge, Kai/Sighard Neckel/Irene Somm (2006). Das Leistungsprinzip als Deutungsressource. Zur Rekonstruktion von gesellschaftlichem Bewertungswissen, in: Ralf Bohnsack/Aglaja Przyborski/Burkhard Schäffer (Hg.): Das Gruppendiskussionsverfahren in der Forschungspraxis, Opladen, 203-215.

Dröge, Kai/Irene Somm (2005). Spurlose Leistung. Langsicht im flexiblen Kapitalismus, in: bios, Vol. I8(2), 215-235.

Dubet, François/Didier Lapeyronnie (1994). Im Aus der Vorstädte. Der Zerfall der demokratischen Gesellschaft, Stuttgart.

Durkheim, Émile (1973). Der Selbstmord, Neuwied/Berlin.

Durkheim, Émile (1992). Über soziale Arbeitsteilung. Studie über die Organisation höherer Gesellschaften, Frankfurt am Main.

Goffman, Erving (1976). Frame Analysis. An Essay on the Organization of Experience, Cambridge.

Hartfiel, Günter (1977). Einleitung, in: Günter Hartfiel (Hg.): Das Leistungsprinzip. Merkmale - Bedingungen - Probleme, Opladen, 7-48.

Honneth, Axel (1994). Kampf um Anerkennung. Zur moralischen Grammatik sozialer Konflikte, Frankfurt am Main.

Honneth, Axel (2002). Organisierte Selbstverwirklichung. Paradoxien der Individualisierung, in: Axel Honneth (Hg.): Befreiung aus der Mündigkeit. Paradoxien des gegenwärtigen Kapitalismus, Frankfurt am Main/New York, I4I-I58.

Honneth, Axel (2003). Umverteilung als Anerkennung. Eine Erwiderung auf Nancy Fraser, in: Nancy Fraser/Axel Honneth: Umverteilung oder Anerkennung? Eine politisch-philosophische Kontroverse, Frankfurt am Main, I29-224.
Honneth, Axel (2008). Arbeit und Anerkennung. Versuch einer Neubestimmung, in: Deutsche Zeitschrift für Philosophie, Vol. 56(3), 327-34I.

Honneth, Axel (20II). Das Recht der Freiheit. Grundriß einer demokratischen Sittlichkeit, Berlin.

Hürtgen, Stefanie/Stephan Voswinkel (2014). Nichtnormale Normalität? Anspruchslogiken aus der Arbeitnehmermitte, Berlin.

Jahoda, Marie/Paul F. Lazarsfeld/Hans Zeisel (1975). Die Arbeitslosen von Marienthal. Ein soziographischer Versuch über die Wirkungen langandauernder Arbeitslosigkeit, Frankfurt am Main.

Kleemann, Frank/Ingo Matuschek/Günter Voß (2002). Subjektivierung von Arbeit - Ein Überblick zum Stand der soziologischen Diskussion, in: Manfred Moldaschl/G. Günter Voß (Hg.): Subjektivierung der Arbeit, München, 53-IOO.

Kocyba, Hermann (2000). Der Preis der Anerkennung: Von der tayloristischen Mißachtung zur strategischen Instrumentalisierung der Subjektivität der Arbeitenden, in: Ursula Holtgrewe/Stephan Voswinkel/Gabriele Wagner (Hg.): Anerkennung und Arbeit, Konstanz, I27-I40.

Mayntz, Renate (196I). Kritische Bemerkungen zur funktionalistischen Schichtungstheorie, in: David V. Glass/Réne König (Hg.): Soziale Schichtung und soziale Mobilität. Kölner Zeitschrift für Soziologie und Sozialpsychologie, Sonderheft 5, Opladen, IO-28.

Mead, George Herbert (1973). Geist, Identität und Gesellschaft aus der Sicht des Sozialbehaviorismus, Frankfurt am Main.

Menz, Wolfgang (2009). Die Legitimität des Marktregimes. Leistungs- und Gerechtigkeitsorientierungen in neuen Formen betrieblicher Leistungspolitik, Wiesbaden.

Moldaschl, Manfred/G. Günter Voß (Hg.) (2002). Subjektivierung der Arbeit, München.

Neckel, Sighard/Kai Dröge (2002). Die Verdienste und ihr Preis: Leistung in der Marktgesellschaft, in: Axel Honneth (Hg.): Befreiung aus der Mündigkeit. Paradoxien des gegenwärtigen Kapitalismus, Frankfurt am Main/New York, 93-II6.

Neckel, Sighard/Kai Dröge/Irene Somm (2004). Welche Leistung, welche Leistungsgerechtigkeit? Soziologische Konzepte, normative Fragen und einige empirische Befunde, in: Peter A. Berger/Volker H. Schmidt (Hg.): Welche Gleichheit, welche Ungleichheit? Grundlagen der Ungleichheitsforschung, Wiesbaden, I37-I64.

Neckel, Sighard/Kai Dröge/Irene Somm (2005). Das umkämpfte Leistungsprinzip. Deutungskonflikte um die Legitimationen sozialer Ungleichheit, in: WSIMitteilungen, Vol. 58(7), 368-374.

Noelle-Neumann, Elisabeth (1978). Werden wir alle Proletarier? Wertewandel in unserer Gesellschaft, Zürich. 
Offe, Claus (1970). Leistungsprinzip und industrielle Arbeit. Mechanismen der Statusverteilung in Arbeitsorganisationen der industriellen „Leistungsgesellschaft", Frankfurt am Main.

Paulus, Stefan (2013). Hausarbeitsdebatte revisited. Zur Arbeitswerttheorie von Haus- und Reproduktionsarbeit. Internet: http://doku.b.tu-harburg.de/volltexte/2OI3/1203 (Zugriff: 17.7.2015).

Popitz, Heinrich/Hans Paul Bahrdt/Ernst August Jüres/ Hanno Kesting (1957). Das Gesellschaftsbild des Arbeiters. Soziologische Untersuchungen in der Hüttenindustrie, Tübingen.

Schroeder, W. (Hg.) (20I4). Handbuch Gewerkschaften in Deutschland, Wiesbaden.

Todorov, Tzvetan (1996). Abenteuer des Zusammenlebens. Versuch einer allgemeinen Anthropologie, Berlin.

Tumin, Melvin M. (1966). Some Principles of Stratification. A Critical Analysis, in: Reinhard Bendix/Seymour Martin Lipset (Hg.): Class, Status, and Power. Social Stratification in Comparative Perspective (Second Edition), New York, 53-58.

Voswinkel, Stephan (2002). Bewunderung ohne Würdigung? Paradoxien der Anerkennung doppelt subjektivierter Arbeit, in: Axel Honneth (Hg.): Befreiung aus der Mündigkeit. Paradoxien des gegenwärtigen Kapitalismus, Frankfurt am Main/New York, 65-92.

Voswinkel, Stephan/Hermann Kocyba (2008). Die Kritik des Leistungsprinzips im Wandel, in: Kai Dröge/Kira Marrs/Wolfgang Menz (Hg.): Rückkehr der Leistungsfrage. Leistung in Arbeit, Unternehmen und Gesellschaft, Berlin, 2I-39.

Voswinkel, Stephan/Anna Korzekwa (2005). Welche Kundenorientierung? Anerkennung in der Dienstleistungsarbeit, Berlin.

Wagner, Gabriele (2008). „Ausschließlich zahlenorientiert, ausschließlich an Erträgen orientiert“. Vermarktlichung als Organisationsprinzip und Anerkennungsproblem, in: Österreichische Zeitschrift für Soziologie, Vol. 33(3), 20-42.

Weber, Max (1988). Die protestantische Ethik und der Geist des Kapitalismus, in: Ders.: Gesammelte Aufsätze zur Religionssoziologie, Tübingen, 17-206.

Wittig-Koppe, Holger/Bremer, Fritz, Hansen, Hartwig (Hg.) (20IO). Teilhabe in Zeiten verschärfter Ausgrenzung? Kritische Beiträge zur Inklusiondebatte, Neumünster.

\section{Autor}

Dr. Kai Dröge, Wissenschaftlicher Mitarbeiter am Institut für Sozialforschung (Frankfurt am Main) sowie Dozent und Modulleiter an der Hochschule Luzern Wirtschaft. Forschungsschwerpunkte: Soziologie des Ökonomischen; Kultur- und Mediensoziologie; Paarsoziologie; Soziale Ungleichheit, Gerechtigkeitsforschung 